\title{
Study of Heterosis for Yield and Yield Contributing Traits in Desi Cotton (Gossypium arboreum L.)
}

\author{
A.V. Shinde ${ }^{1}$, D.B. Deosarkar ${ }^{2}$, V.N. Chinchane ${ }^{3 *}$, A.S. Kalambe ${ }^{4}$, N. Harshika ${ }^{5}$ \\ Department of Agricultural Botany, VNMKV, Parbhani, India \\ *Corresponding author:
}

\section{A B S T R A C T}

\section{Keywords}

Heterosis, Cotton, Randomized Block Design, Yield

Article Info

Accepted:

22 July 2018

Available Online:

10 August 2018
The Line $\mathrm{x}$ Tester method of analysis was followed involving 5 females viz., PA 740, PA760, PA848, PA828 and PAIG 77 and 6 males viz., AKA 9703, JLA 505, RAC 024, AKA 7, PA 08 and Phule Dhanwantary for study of heterosis for various yield and fibre characters. The $\mathrm{F}_{1}$ 's and their parents were evaluated in Randomized Block Design with two replications. Observations were recorded on Days to $50 \%$ flowering, Plant height (cm), Number of sympodia per plant, Number of bolls per plant, Boll weight $(\mathrm{g})$, Seed index, Seed cotton yield per plant $(\mathrm{g})$ and Lint index. The high magnitude of heterosis for seed cotton yield per plant indicated that the cross PA 848 x Phule Dhanwantary (118.59 \%), PAIG 77 x AKA 9703 (93.34 \%) and PA 760 x PA 08 (82.22\%).

\section{Introduction}

Cotton is the most important fibre crop of India. Despite the increasing production of synthetic fibre, cotton has its reputation as "King of Fibres" due to its inherent properties. The production of cotton in the country is not making a striding increase. Conversely, the yield plateau in the cotton productivity can be broken by identifying high economic heterosis. The low production of cotton can be increased by increasing the area under hybrid cultivation, as hybrids are not only important for their high productivity but are generally good for stability for production also. They hold the key for making breakthrough in production of cotton and therefore, should be given more attention. For commercial exploitation of heterosis, the magnitude of heterosis provides a basis for genetic diversity and is a guide to the choice of desirable parents for developing superior F1hybrids, so as to exploit hybrid vigour or building the better gene pool after growing in subsequent generations. Cotton improvement programmes primarily lay emphasis from development of hybrids, which have contributed in improving productivity of cotton (Christopher et al, 2003). Hybridization is the most potent technique for breaking yield barriers. Effective improvement in yield may be brought about through selection on yield component 
characters. The objective of the present study was to determine the extent of heterosis foe seed cotton yield and yield contributing traits to identify promising hybrids.

\section{Materials and Methods}

The present investigation on "study of heterosis for yield and fibre quality traits in desi cotton" was conducted at Cotton Research Station, Mahboob Baugh Farm, VNMKV, Parbhani.

The experimental material for the present investigation consisted of eleven diverse genotypes. These selected eight genotypes possess good amount of variation for seed cotton yield per plant along with yield contributing traits were, Lines: PA 740, PA 760, PA 848, PA 828 and PAIG 77, Testers: AKA 9703, JLA 505, RAC 024, AKA 7 PA 08 and Phule Dhanwantary.

The experiment was laid in Randomized Block Design with two replications. The treatment consists of thirty crosses $\left(F_{1} s\right)$ and eleven parents in the experiment.

The variety PKVDH 1, PKV Suvarna and PhuleDhanwantary were used as checks and parents were sown in adjacent separate blocks. Each treatment was randomized in each block sown in rows of $60 \mathrm{~cm} \times 30 \mathrm{~cm}$ spacing. The heterosis was calculated over mid parent, better parent and standard checks as per the procedure suggested by Fonesca and Patterson (1968).

\section{Results and Discussion}

The analysis of variance showed significant differences among treatments for the characters studied (Table 1).

Heterosis (\%) over mid parent (MP), better parent (BP) and standard check (SC) was calculated for yield and yield contributing characters. The results obtained are presented in Table 2, which are discussed below.

For days to 50 per cent flowering, the cross combination PA 848 x PA 08 displayed highest significant negative heterosis over mid parent (- $15.79 \%)$, PA 828 x AKA 7 ($15.89 \%$ ), while the cross combination PA 828 $\mathrm{X}$ AKA $7(-11.19 \%)$ recorded significant negative heterosis over standard check PKVDH 1 and NACH 12. Out of thirty crosses, four crosses exhibited negatively significant heterosis over standard check PKV Suvarna. Significant negative heterosis for earliness was also reported by Deosarkar et al., (2009), Patel et al., (2010) and Jaiwar et al., (2012).

High number of sympodia per plant with minimum number of monopodial branches is an indication of higher productivity. The cross combination PA 848 x JLA 505 exhibited highest significant positive heterosis over mid parent and better parent, whereas cross combination PAIG 77 x Phule Dhanwantary displayed highest positive significant heterosis over standard check PKV Suvarna. Seventeen crosses were found significantly superior over standard check PKV Suvarna. Heterosis for this trait was also reported by the earlier workers Tuteja et al., (2011) and Balu et al., (2012).

Plant height is an important morphological trait in cotton which provides space for nodes and internodes from where monopodial and sympodial branches arise. Thus it plays an important role in determining the morphological frame work relating to plant type, duration and productivity. The proportion of sympodial and monopodial branches, size and arrangement in a particular genotype together with height of the plant determine architecture of the cotton plant. 
Table.1 Analysis of variance for Randomized Block Design

\begin{tabular}{|c|c|c|c|c|c|c|c|c|c|c|}
\hline $\begin{array}{l}\text { Source of } \\
\text { variation }\end{array}$ & d.f. & $\begin{array}{c}\text { Days to } \\
50 \% \\
\text { flowering }\end{array}$ & $\begin{array}{c}\text { No. of } \\
\text { sympodia/plant }\end{array}$ & $\begin{array}{c}\text { No. of } \\
\text { bolls/plant }\end{array}$ & $\begin{array}{c}\text { Boll } \\
\text { weight } \\
\text { (g) }\end{array}$ & $\begin{array}{c}\text { Plant } \\
\text { height } \\
\text { (cm) }\end{array}$ & $\begin{array}{l}\text { Days to } \\
\text { maturity }\end{array}$ & $\begin{array}{c}\text { Seed } \\
\text { cotton } \\
\text { yield } \\
\text { /plant }(\mathrm{g})\end{array}$ & $\begin{array}{l}\text { Lint } \\
\text { index }\end{array}$ & $\begin{array}{c}\text { Seed } \\
\text { index } \\
(\mathrm{g})\end{array}$ \\
\hline Replications & 1 & 15.55 & 0.275 & 6.545 & 0.004 & 44.84 & 12.37 & 0.936 & 0.007 & 0.036 \\
\hline Treatments & 43 & $23.76 * *$ & $3.943 * *$ & $16.37 * *$ & $0.045^{* *}$ & $997.64 * *$ & $44.01 * *$ & $122.89 * *$ & $0.046 * *$ & $1.352 * *$ \\
\hline Error & 43 & 7.25 & 0.444 & 2.824 & 0.021 & 15.26 & 7.351 & 5.731 & 0.019 & 0.200 \\
\hline
\end{tabular}

\begin{tabular}{|c|c|c|c|c|c|c|}
\hline $\begin{array}{c}\text { Source of } \\
\text { variation }\end{array}$ & d.f. & $\begin{array}{l}\text { Ginning out } \\
\text { turn }(\%)\end{array}$ & $\begin{array}{c}\text { Upper half } \\
\text { mean length } \\
(\mathrm{mm})\end{array}$ & $\begin{array}{c}\text { Fibre fineness } \\
\text { (micronaire) } \\
(\mu \mathrm{g} / \mathrm{inch})\end{array}$ & $\begin{array}{l}\text { Fibre strength } \\
(\mathrm{g} / \mathrm{tex})\end{array}$ & $\begin{array}{c}\text { Uniformity } \\
\text { ratio }(\%)\end{array}$ \\
\hline & \multicolumn{6}{|c|}{ Mean sum of squares } \\
\hline Replications & 1 & 1.550 & 2.635 & 0.045 & 3.180 & 2.556 \\
\hline Treatments & 43 & $35.45 * *$ & $5.545 * *$ & $0.162 * *$ & $4.701 * *$ & $4.138 * *$ \\
\hline Error & 43 & 1.481 & 1.457 & 0.039 & 1.252 & 1.859 \\
\hline
\end{tabular}

$*$, ** significant at $5 \%$ and $1 \%$ levels, respectively 
Table.2 Estimates of heterosis in percentage over mid parent (M.P.), better parent (B.P.) and standard checks (S.C.) for yield and yield contributing characters

\begin{tabular}{|c|c|c|c|c|c|c|c|c|c|c|c|c|c|}
\hline \multirow{3}{*}{$\begin{array}{l}\mathrm{Sr} \\
\text { no }\end{array}$} & \multirow[t]{3}{*}{ Hybrids } & \multicolumn{6}{|c|}{ Days to $50 \%$ flowering } & \multicolumn{6}{|c|}{ No. of sympodia/plant } \\
\hline & & \multirow[t]{2}{*}{ Mean } & \multirow{2}{*}{$\begin{array}{c}\text { M.P. } \\
\text { Heterosis } \\
(\%)\end{array}$} & \multirow{2}{*}{$\begin{array}{c}\text { B.P. } \\
\text { Heterosis } \\
(\%)\end{array}$} & \multicolumn{3}{|c|}{ \% standard heterosis over } & \multirow[t]{2}{*}{ Mean } & \multirow{2}{*}{$\begin{array}{c}\text { M.P. } \\
\text { Heterosis } \\
(\%)\end{array}$} & \multirow{2}{*}{$\begin{array}{c}\text { B.P. } \\
\text { Heterosis } \\
(\%)\end{array}$} & \multicolumn{3}{|c|}{ \% standard heterosis over } \\
\hline & & & & & PKVDH1 & $\begin{array}{c}\text { PKV } \\
\text { Suvarna }\end{array}$ & $\begin{array}{c}\text { NACH } \\
12\end{array}$ & & & & PKVDH1 & $\begin{array}{c}\text { PKV } \\
\text { Suvarna }\end{array}$ & NACH 12 \\
\hline 1 & PA $740 \times$ AKA 9703 & 71.50 & -3.70 & -5.92 & 0.00 & -2.72 & 0.70 & 14.74 & 8.60 & -7.47 & 3.55 & $25.86^{* *}$ & -0.94 \\
\hline 2 & PA 740 x JLA 505 & 72.00 & -3.03 & -5.26 & 0.70 & -2.04 & 1.41 & 12.91 & $-10.05^{*}$ & $-18.95^{* *}$ & -9.30 & 10.24 & $-13.23 * *$ \\
\hline 3 & PA $740 \times$ RAC 024 & 70.50 & -3.75 & -4.73 & -1.40 & -4.08 & -0.70 & 11.25 & $-16.46^{* *}$ & $-29.43 * *$ & $-21.03 * *$ & -4.01 & $-24.45^{* *}$ \\
\hline 4 & PA $740 \times$ AKA 7 & 76.00 & 5.19 & 4.83 & 6.29 & 3.40 & 7.04 & 12.82 & $-8.95^{*}$ & $-19.58 * *$ & $-10.01 *$ & 9.39 & $-13.91 * *$ \\
\hline 5 & PA $740 \times$ PA 08 & 71.50 & -3.70 & -5.92 & 0.00 & -2.72 & 0.70 & 13.94 & 1.79 & $-12.49 * *$ & -2.07 & $19.04 * *$ & -6.32 \\
\hline 6 & PA 740 x PhuleDhanwantary & 74.50 & 3.11 & 2.76 & 4.20 & 1.36 & 4.93 & 13.28 & -2.83 & $-16.63 * *$ & -6.71 & $13.40^{*}$ & $-10.75^{*}$ \\
\hline 7 & PA $760 \times$ AKA 9703 & 75.00 & -0.66 & -1.32 & 4.90 & 2.04 & 5.63 & 14.49 & $11.84 *$ & -1.39 & 1.79 & $23.73 * *$ & -2.62 \\
\hline 8 & PA 760 x JLA 505 & 75.00 & -0.66 & -1.32 & 4.90 & 2.04 & 5.63 & 12.45 & $-9.43 *$ & $-15.34 * *$ & $-12.61 *$ & 6.23 & $-16.39 * *$ \\
\hline 9 & PA $760 \times$ RAC 024 & 78.00 & 4.70 & 4.00 & $9.09 *$ & 6.12 & $9.86^{*}$ & 13.01 & 1.30 & $-11.50^{*}$ & -8.64 & 11.05 & $-12.60 * *$ \\
\hline 10 & PA $760 \times$ AKA 7 & 72.50 & -1.36 & -3.33 & 1.40 & -1.36 & 2.11 & 12.22 & $-9.20 *$ & $-16.87 * *$ & $-14.19 * *$ & 4.31 & $-17.90 * *$ \\
\hline 11 & PA $760 \times$ PA 08 & 70.50 & $-6.62 *$ & -7.24 & -1.40 & -4.08 & -0.70 & 11.07 & $-15.42 * *$ & $-24.73 * *$ & $-22.30 * *$ & -5.55 & $-25.66^{* *}$ \\
\hline 12 & PA 760 x PhuleDhanwantary & 74.50 & 1.36 & -0.67 & 4.20 & 1.36 & 4.93 & 13.52 & 3.56 & -8.03 & -5.06 & $15.41 *$ & -9.17 \\
\hline 13 & PA $848 \times$ AKA 9703 & 68.50 & $-9.87 * *$ & $-9.87 *$ & -4.20 & -6.80 & -3.52 & 11.72 & -7.15 & $-16.46^{* *}$ & $-17.66^{* *}$ & 0.09 & $-21.23 * *$ \\
\hline 14 & PA 848 x JLA 505 & 72.00 & -5.26 & -5.26 & 0.70 & -2.04 & 1.41 & 10.30 & $-23.21 * *$ & $-26.65 * *$ & $-27.70 * *$ & $-12.12 *$ & $-30.84 * *$ \\
\hline 15 & PA $848 \times$ RAC 024 & 73.00 & -2.67 & -3.95 & 2.10 & -0.68 & 2.82 & 15.16 & $21.22 * *$ & 8.05 & 6.50 & $29.45 * *$ & 1.88 \\
\hline 16 & PA $848 \times$ AKA 7 & 65.00 & $-12.16^{* *}$ & $-14.47 * *$ & $-9.09 *$ & $-11.56^{* *}$ & $-8.45^{*}$ & 12.19 & -7.09 & $-13.11 *$ & $-14.36^{* *}$ & 4.10 & $-18.07 * *$ \\
\hline 17 & PA $848 \times$ PA 08 & 64.00 & $-15.79 * *$ & $-15.79 * *$ & $-10.49 *$ & $-12.93 * *$ & $-9.86^{*}$ & 11.67 & -8.47 & $-16.85^{* *}$ & $-18.05 * *$ & -0.38 & $-21.60 * *$ \\
\hline 18 & PA 848 x PhuleDhanwantary & 72.00 & -2.70 & -5.26 & 0.70 & -2.04 & 1.41 & 14.04 & $10.36^{*}$ & 0.04 & -1.40 & $19.85 * *$ & -5.68 \\
\hline 19 & PA 828 x AKA 9703 & 76.00 & 0.33 & 0.00 & 6.29 & 3.40 & 7.04 & 13.13 & 3.90 & -6.58 & -7.79 & $12.08 *$ & $-11.79 *$ \\
\hline 20 & PA 828 x JLA 505 & 68.00 & $-10.23 * *$ & $-10.53 * *$ & -4.90 & -7.48 & -4.23 & 11.21 & $-16.45^{* *}$ & $-20.24 * *$ & $-21.28 * *$ & -4.31 & $-24.69 * *$ \\
\hline 21 & PA $828 \times$ RAC 024 & 68.50 & $-8.36^{*}$ & $-9.27 *$ & -4.20 & -6.80 & -3.52 & 13.58 & 8.47 & -3.38 & -4.63 & $15.92 * *$ & -8.77 \\
\hline 22 & PA $828 \times$ AKA 7 & 63.50 & $-13.90 * *$ & $-15.89 * *$ & $-11.19 * *$ & $-13.61 * *$ & $-10.56^{*}$ & 13.55 & 3.12 & -3.63 & -4.88 & $15.62 *$ & -9.00 \\
\hline 23 & PA $828 \times$ PA 08 & 70.00 & $-7.59^{*}$ & $-7.89 *$ & -2.10 & -4.76 & -1.41 & 13.26 & 3.88 & -5.69 & -6.92 & $13.15^{*}$ & $-10.95^{*}$ \\
\hline 24 & PA 828 x PhuleDhanwantary & 66.50 & $-9.83 * *$ & $-11.92 * *$ & -6.99 & $-9.52 *$ & -6.34 & 13.62 & 6.97 & -3.09 & -4.35 & $16.26 * *$ & -8.50 \\
\hline 25 & PAIG 77 x AKA 9703 & 72.50 & -4.92 & -5.23 & 1.40 & -1.36 & 2.11 & 14.39 & $10.44 *$ & -3.00 & 1.02 & $22.79 * *$ & -3.36 \\
\hline 26 & PAIG 77 x JLA 505 & 74.50 & -2.30 & -2.61 & 4.20 & 1.36 & 4.93 & 14.28 & 3.44 & -3.71 & 0.28 & $21.90 * *$ & -4.06 \\
\hline 27 & PAIG 77 x RAC 024 & 75.50 & 0.33 & -1.31 & 5.59 & 2.72 & 6.34 & 13.94 & 8.00 & -6.00 & -2.11 & $18.99 * *$ & -6.35 \\
\hline 28 & PAIG 77 x AKA 7 & 75.00 & 1.01 & -1.96 & 4.90 & 2.04 & 5.63 & 14.54 & 7.49 & -1.99 & 2.07 & $24.07 * *$ & -2.35 \\
\hline 29 & PAIG 77 x PA 08 & 74.00 & -2.95 & -3.27 & 3.50 & 0.68 & 4.23 & 11.95 & -9.07 & $-19.39 * *$ & $-16.05 * *$ & 2.05 & $-19.68 * *$ \\
\hline \multirow[t]{2}{*}{30} & PAIG 77 x PhuleDhanwantary & 69.00 & $-7.07 *$ & $-9.80 *$ & -3.50 & -6.12 & -2.82 & 15.33 & $16.84 * *$ & 3.37 & 7.65 & $30.86 * *$ & 2.99 \\
\hline & S.E. \pm & 72.42 & 2.406 & 2.778 & 2.778 & 2.778 & 2.778 & 13.13 & 0.583 & 0.673 & 0.673 & 0.673 & 0.673 \\
\hline $\mathrm{Sr}$ & Hybrids & \multicolumn{6}{|c|}{ No. of bolls/plant } & \multicolumn{5}{|c|}{ Boll weight (g) } & \\
\hline
\end{tabular}




\begin{tabular}{|c|c|c|c|c|c|c|c|c|c|c|c|c|c|}
\hline \multirow[t]{2}{*}{ no } & & \multirow[t]{2}{*}{ Mean } & \multirow{2}{*}{$\begin{array}{c}\text { M.P. } \\
\text { Heterosis } \\
(\%)\end{array}$} & \multirow{2}{*}{$\begin{array}{c}\text { B.P. } \\
\text { Heterosis } \\
(\%)\end{array}$} & \multicolumn{3}{|c|}{ \% standard heterosis over } & \multirow[t]{2}{*}{ Mean } & \multirow{2}{*}{$\begin{array}{c}\text { M.P. } \\
\text { Heterosis } \\
(\%)\end{array}$} & \multirow{2}{*}{$\begin{array}{c}\text { B.P. } \\
\text { Heterosis } \\
(\%)\end{array}$} & \multicolumn{3}{|c|}{ \% standard heterosis over } \\
\hline & & & & & PKVDH1 & $\begin{array}{c}\text { PKV } \\
\text { Suvarna }\end{array}$ & NACH 12 & & & & PKVDH1 & $\begin{array}{c}\text { PKV } \\
\text { Suvarna }\end{array}$ & NACH 12 \\
\hline 1 & PA $740 \times$ AKA 9703 & 18.50 & 7.25 & 0.00 & 15.63 & -5.13 & -15.91 & 2.50 & $17.55^{* *}$ & $17.14^{*}$ & 10.89 & 12.13 & 1.22 \\
\hline 2 & PA 740 x JLA 505 & 16.00 & 0.00 & 0.00 & 0.00 & -17.95 & $-27.27 * *$ & 2.20 & 4.27 & 3.29 & -2.22 & -1.12 & -10.75 \\
\hline 3 & PA $740 \times$ RAC 024 & 22.50 & $45.16^{* *}$ & $40.63 * *$ & $40.63 * *$ & 15.38 & 2.27 & 2.73 & $31.01 * *$ & $27.93 * *$ & $21.11 * *$ & $22.47 * *$ & 10.55 \\
\hline 4 & PA $740 \times$ AKA 7 & 18.50 & -3.90 & $-17.78 *$ & 15.63 & -5.13 & -15.91 & 2.22 & 0.91 & -2.20 & -1.33 & -0.22 & -9.94 \\
\hline 5 & PA $740 \times$ PA 08 & 17.00 & 4.62 & 3.03 & 6.25 & -12.82 & $-22.73 * *$ & 2.15 & -1.15 & -3.15 & -4.44 & -3.37 & $-12.78^{*}$ \\
\hline 6 & PA $740 \times$ PhuleDhanwantary & 16.50 & 3.13 & 3.13 & 3.13 & -15.38 & $-25.00 * *$ & 2.31 & 5.48 & 2.67 & 2.67 & 3.82 & -6.29 \\
\hline 7 & PA $760 \times$ AKA 9703 & 19.00 & 15.15 & 2.70 & 18.75 & -2.56 & -13.64 & 2.13 & 2.77 & 0.71 & -5.33 & -4.27 & $-13.59 *$ \\
\hline 8 & PA 760 x JLA 505 & 18.50 & $21.31^{*}$ & 15.63 & 15.63 & -5.13 & -15.91 & 2.25 & 9.22 & 7.66 & 0.00 & 1.12 & -8.72 \\
\hline 9 & PA $760 \times$ RAC 024 & 18.50 & $25.42 *$ & 23.33 & 15.63 & -5.13 & -15.91 & 2.24 & 1.10 & 10.10 & -0.67 & 0.45 & -9.33 \\
\hline 10 & PA $760 \times$ AKA 7 & 18.00 & -2.70 & $-20.00^{*}$ & 12.50 & -7.69 & $-18.18^{*}$ & 2.02 & -6.05 & -11.01 & -10.22 & -9.21 & $-18.05 * *$ \\
\hline 11 & PA $760 \times$ PA 08 & 24.00 & $-54.84 * *$ & $45.45^{* *}$ & $50.00 *$ & $23.08^{*}$ & 9.09 & 2.32 & 9.41 & 4.73 & 3.33 & 4.49 & -5.68 \\
\hline 12 & PA 760 x PhuleDhanwantary & 20.00 & $31.15^{* *}$ & $25.00 *$ & $25.00 *$ & 2.56 & -9.09 & 2.64 & $23.60 * *$ & $17.56^{*}$ & $17.56^{*}$ & $18.88 * *$ & 7.30 \\
\hline 13 & PA $848 \times$ AKA 9703 & 25.00 & $44.93 * *$ & $35.14 * *$ & $56.25 * *$ & $28.21 * *$ & 13.64 & 2.16 & -4.10 & -9.79 & -3.78 & -2.70 & -12.17 \\
\hline 14 & PA 848 x JLA 505 & 16.00 & 0.00 & 0.00 & 0.00 & -17.95 & $-27.27 * *$ & 2.00 & -11.14 & $-16.88^{*}$ & -11.33 & -10.34 & $-19.07 * *$ \\
\hline 15 & PA $848 \times$ RAC 024 & 17.00 & 9.68 & 6.25 & 6.25 & -12.82 & $-22.73 * *$ & 2.07 & -6.55 & $-13.75^{*}$ & -8.00 & -6.97 & $-16.02 *$ \\
\hline 16 & PA $848 \times$ AKA 7 & 17.00 & -11.69 & $-24.44 * *$ & 6.25 & -12.82 & $-22.73 * *$ & 2.13 & -8.99 & -11.46 & -5.56 & -4.49 & $-13.79 *$ \\
\hline 17 & PA $848 \times$ PA 08 & 20.00 & $23.08 *$ & 21.21 & $25.00 *$ & 2.56 & -9.09 & 2.15 & -6.71 & -10.21 & -4.22 & -3.15 & $-12.58 *$ \\
\hline 18 & PA $848 \times$ PhuleDhanwantary & 26.00 & $62.50 * *$ & $62.50 * *$ & $62.50 * *$ & $33.33^{* *}$ & $18.18^{*}$ & 2.24 & -3.66 & -6.67 & -0.44 & 0.67 & -9.13 \\
\hline 19 & PA $828 \times$ AKA 9703 & 19.50 & 16.42 & 5.41 & 21.88 & 0.00 & -11.36 & 2.22 & 3.13 & 1.14 & -1.11 & 0.00 & -9.74 \\
\hline 20 & PA 828 x JLA 505 & 21.00 & $35.48 * *$ & $31.25 * *$ & $31.25 * *$ & 7.69 & -4.55 & 2.25 & 5.13 & 2.50 & 0.22 & 1.35 & -8.52 \\
\hline 21 & PA $828 \times$ RAC 024 & 21.50 & $43.33 * *$ & $43.33 * *$ & $34.38 * *$ & 10.26 & -2.27 & 2.29 & 8.27 & 4.09 & 1.78 & 2.92 & -7.10 \\
\hline 22 & PA $828 \times$ AKA 7 & 14.00 & $-37.78 * *$ & $-37.78 * *$ & -12.50 & $-28.21 * *$ & $-36.36 * *$ & 2.27 & 1.57 & 0.00 & 0.89 & 2.02 & -7.91 \\
\hline 23 & PA $828 \times$ PA 08 & 17.50 & 6.06 & 6.06 & 9.38 & -10.26 & $-20.45^{*}$ & 2.03 & -8.37 & -8.78 & -10.00 & -8.99 & $-17.85 * *$ \\
\hline 24 & PA $828 \times$ PhuleDhanwantary & 19.50 & 21.88 & 21.88 & 21.88 & 0.00 & -11.36 & 2.30 & 3.15 & 2.00 & 2.00 & 3.15 & -6.90 \\
\hline 25 & PAIG 77 x AKA 9703 & 23.00 & $24.32 *$ & $24.32 *$ & $43.75 * *$ & 17.95 & 4.55 & 2.08 & -2.00 & -2.35 & -7.56 & -6.52 & $-15.62 *$ \\
\hline 26 & PAIG 77 x JLA 505 & 20.00 & $25.00 *$ & $25.00^{*}$ & $25.00 *$ & 2.56 & -9.09 & 2.22 & 5.21 & 4.23 & -1.33 & -0.22 & -9.94 \\
\hline 27 & PAIG 77 x RAC 024 & 19.00 & $26.67 *$ & $26.67 *$ & 18.75 & -2.56 & -13.64 & 2.29 & 10.10 & 7.51 & 1.78 & 2.92 & -7.10 \\
\hline 28 & PAIG 77 x AKA 7 & 18.00 & $-20.00 *$ & $-20.00 *$ & 12.50 & -7.69 & $-18.18^{*}$ & 2.17 & -1.59 & -4.63 & -3.78 & -2.70 & -12.17 \\
\hline 29 & PAIG 77 x PA 08 & 17.00 & 3.03 & 3.03 & 6.25 & -12.82 & $-22.73 * *$ & 2.16 & -0.69 & -2.70 & -4.00 & -2.92 & -12.37 \\
\hline \multirow[t]{2}{*}{30} & PAIG 77 x PhuleDhanwantary & 16.00 & 0.00 & 0.00 & 0.00 & -17.95 & $-27.27 * *$ & 2.17 & -0.91 & -3.56 & -3.56 & -2.47 & -11.97 \\
\hline & S.E. \pm & 18.50 & 1.488 & 1.718 & 1.718 & 1.718 & 1.718 & 2.220 & 0.129 & 0.149 & 0.149 & 0.149 & 0.149 \\
\hline
\end{tabular}




\begin{tabular}{|c|c|c|c|c|c|c|c|c|c|c|c|c|c|}
\hline \multirow[t]{3}{*}{ Sr no } & \multirow[t]{3}{*}{ Hybrids } & \multicolumn{6}{|c|}{ Plant height $(\mathrm{cm})$} & \multicolumn{6}{|c|}{ Seed cotton yield/plant (g) } \\
\hline & & \multirow[t]{2}{*}{ Mean } & \multirow{2}{*}{$\begin{array}{c}\text { M.P. } \\
\text { Heterosis } \\
(\%)\end{array}$} & \multirow{2}{*}{$\begin{array}{c}\text { B.P. } \\
\text { Heterosis } \\
(\%)\end{array}$} & \multicolumn{3}{|c|}{$\%$ standard heterosis over } & \multirow[t]{2}{*}{ Mean } & \multirow{2}{*}{$\begin{array}{c}\text { M.P. } \\
\text { Heterosis } \\
(\%)\end{array}$} & \multirow{2}{*}{$\begin{array}{c}\text { B.P. } \\
\text { Heterosis } \\
(\%)\end{array}$} & \multicolumn{3}{|c|}{ \% standard heterosis over } \\
\hline & & & & & PKVDH1 & $\begin{array}{c}\text { PKV } \\
\text { Suvarna }\end{array}$ & $\begin{array}{c}\text { NACH } \\
12\end{array}$ & & & & PKVDH1 & $\begin{array}{c}\text { PKV } \\
\text { Suvarna }\end{array}$ & NACH 12 \\
\hline 1 & PA 740 x AKA 9703 & 131.13 & $-22.20 * *$ & $-31.33 * *$ & $-17.49 * *$ & $-7.56^{*}$ & $-10.82 * *$ & 32.15 & $42.60 * *$ & $36.32 * *$ & $36.81 * *$ & 3.88 & $-21.20 * *$ \\
\hline 2 & PA 740 x JLA 505 & 123.35 & $-11.15 * *$ & $-15.59 * *$ & $-22.38 * *$ & $-13.04 * *$ & $-16.11 * *$ & 22.69 & 2.24 & -0.85 & -3.43 & $-26.67 * *$ & $-44.38 * *$ \\
\hline 3 & PA $740 \times$ RAC 024 & 137.95 & 1.13 & $-5.61 *$ & $-13.20 * *$ & -2.75 & $-6.18^{*}$ & 36.50 & $59.48 * *$ & $50.39 * *$ & $55.32 * *$ & $17.93 *$ & -10.54 \\
\hline 4 & PA 740 x AKA 7 & 161.55 & $10.28 * *$ & $10.01^{* *}$ & 1.65 & $13.88^{* *}$ & $9.87 * *$ & 33.60 & 4.83 & $-21.13 * *$ & $42.98 * *$ & 8.56 & $-17.65 * *$ \\
\hline 5 & PA $740 \times$ PA 08 & 180.11 & $38.88^{* *}$ & $23.24 * *$ & $13.33^{* *}$ & 26.97 & $22.49 * *$ & 30.80 & $38.77 * *$ & $34.59 * *$ & $31.06^{* *}$ & -0.48 & $-24.51 * *$ \\
\hline 6 & PA 740 x PhuleDhanwantary & 184.38 & $31.17 * *$ & $26.16^{* *}$ & $16.02 * *$ & $29.98^{* *}$ & $25.39^{* *}$ & 23.89 & 5.30 & 0.06 & 1.68 & $-22.79 * *$ & $-41.43 * *$ \\
\hline 7 & PA $760 \times$ AKA 9703 & 177.64 & $-4.20^{*}$ & $-6.97 * *$ & $11.78^{* *}$ & $25.23 * *$ & $20.81 * *$ & 29.80 & $29.61^{* *}$ & $26.35^{*}$ & $26.81 *$ & -3.72 & $-26.96 * *$ \\
\hline 8 & PA 760 x JLA 505 & 143.85 & $-7.62^{* *}$ & $-20.05^{* *}$ & $-9.49 * *$ & 1.40 & -2.17 & 25.70 & 13.49 & 12.28 & 9.36 & $-16.96^{*}$ & $-37.01 * *$ \\
\hline 9 & PA $760 \times$ RAC & 175.61 & $14.56^{* *}$ & -2.39 & $10.50^{* *}$ & $23.80 * *$ & $19.43^{* *}$ & 32.40 & $38.85^{* *}$ & $33.50^{* *}$ & $37.87 * *$ & 4.68 & $.59 * *$ \\
\hline 10 & PA $760 \times$ AKA 7 & 154.05 & $-5.71 *$ & $-14.38 * *$ & -3.07 & & 4.76 & 25.50 & $-21.54 * *$ & & 8.51 & $-17.61^{*}$ & $-37.50 * *$ \\
\hline 11 & PA $760 \times$ PA 08 & 134.53 & $-8.22^{* * *}$ & -25 . & $-15.35 * *$ & .17 & $-8.51 * *$ & 41.70 & $84.17 * *$ & $82.22 * *$ & $77.45^{* *}$ & $34.73 * *$ & 2.21 \\
\hline 12 & PA 760 x PhuleDhanwantary & 151.01 & -4.09 & $-16.06^{* *}$ & -4.98 & $6.45^{*}$ & 2.70 & 24.78 & 7.09 & 3.77 & 5.45 & $-19.94 *$ & $-39.26 * *$ \\
\hline 13 & PA $848 \times$ AKA 9703 & 176. & $-6.91 * *$ & -7.8 & $1.074 * *$ & $24.07 * *$ & $19.70^{* *}$ & 42.90 & $83.26 * *$ & $81.90^{* *}$ & $82.55 * *$ & $38.61 * *$ & \\
\hline 14 & PA $848 \times$ JLA 5 & 175.00 & $33 * *$ & & 10.11 & $23.37 * *$ & 19.0 & 23.53 & 2.0 & & 0.13 & $-23.97 * *$ & $-42.33 * *$ \\
\hline 15 & PA $848 \times$ RAC & 138.53 & $-11.72 * *$ & $-25.99 * *$ & $-12.83 * *$ & -2 & -5.7 & 23.44 & -1.2 & -3 & -0. & $-24.28 * *$ & .56 \\
\hline 16 & PA 848 x AKA 7 & 178.92 & 7.13 & $-4.41^{*}$ & $12.58 * *$ & $26.13 * *$ & 21.6 & 22.68 & $-31.12^{* *}$ & $-46.77 * *$ & $-46.77 * *$ & -3.51 & -44 \\
\hline 17 & PA 8 & 136 & $-9.12^{* *}$ & $-27.07 * *$ & $-14.11 * *$ & -3.77 & $-7.17 *$ & 35.10 & $52.21 * *$ & $51.07 * *$ & 51.0 & 49.3 & \\
\hline 18 & PA 84 & 143.14 & $-11.14 * *$ & $52 * *$ & $-9.93 * *$ & 0.91 & -2.65 & 52.20 & $121.59 * *$ & $118.59 * *$ & $118.59 * *$ & 122. & $27.94 * *$ \\
\hline 19 & PA 82 & 157.55 & & $49^{* *}$ & -0.87 & $11.06^{* *}$ & 7.15 & 31.50 & $35.05 * *$ & & $3.56^{* *}$ & $34.04 * *$ & $-22.79 * *$ \\
\hline 20 & PA 82 & 129.76 & 2. & -1.3 & $-18.35^{* *}$ & $-8.53 * *$ & $-11.75^{* *}$ & 39.60 & $72.34 * *$ & 71.6 & $71.69^{* *}$ & $1 * *$ & -2.94 \\
\hline 21 & PA 82 & 176.81 & $42.63 * *$ & $39.57^{* * *}$ & & $24.64 * *$ & $20.25 * *$ & 41.90 & $77.04 * *$ & $72.64 * *$ & $72.64 * *$ & $78.30^{* * *}$ & 2.70 \\
\hline 22 & PA 82 & 146.01 & $8.93 * *$ & -0.5 & $3.13 * *$ & 2.93 & -0.70 & 23.60 & $-28.14 * *$ & $-44.61 * *$ & $-44.61 * *$ & 0.40 & $-42.17 * *$ \\
\hline 23 & PA $828 \times$ PA 08 & 190.24 & $62.27 * *$ & $56.91^{* *}$ & $19.71 * *$ & $34.11 * *$ & $29.38^{* *}$ & 27.00 & 17.52 & 17.06 & 17.06 & 14.89 & $-33.82 * *$ \\
\hline 24 & PA $828 \times$ PhuleDhanwar & 169.53 & $32.32 * *$ & $25.58^{* *}$ & $6.67^{*}$ & $19.51 * *$ & $15.29^{* *}$ & 36.80 & $56.78^{* *}$ & $54.10^{* *}$ & $54.10^{* *}$ & $56.60^{* *}$ & -9.80 \\
\hline 25 & PAIG 77 x AKA 9703 & 177.17 & $15.96^{* *}$ & $-7.21 * *$ & $11.48^{* *}$ & $24.90^{* *}$ & $20.49^{* *}$ & 45.60 & $95.56^{* *}$ & $93.34^{* *}$ & $93.34 * *$ & $94.04 * *$ & 11.76 \\
\hline 26 & PAIG 77 x JLA 505 & 158.13 & $28.48^{* *}$ & $20.24^{* *}$ & -0.50 & $11.47^{* *}$ & $7.54 * *$ & 25.20 & 9.71 & 10.09 & 9.33 & 7.23 & $-38.24 * *$ \\
\hline 27 & PAIG 77 x RAC 024 & 150.44 & $24.68^{* *}$ & $18.76^{* *}$ & $-5.34 *$ & $6.05 *$ & 2.31 & 27.90 & 17.92 & 14.96 & 14.96 & 18.72 & $-31.62 * *$ \\
\hline 28 & PAIG 77 x AKA 7 & 188.10 & $43.87 * *$ & $28.09^{* *}$ & $18.36^{* *}$ & $32.60 * *$ & $27.92 * *$ & 22.92 & $-30.24 * *$ & $-46.24 * *$ & $-46.24 * *$ & -2.55 & $-43.87 * *$ \\
\hline 29 & PAIG 77 x PA 08 & 143.01 & $25.52^{* *}$ & $24.75^{* *}$ & $-10.01 * *$ & 0.82 & -2.74 & 30.80 & $34.10^{* *}$ & $33.62 * *$ & $33.62 * *$ & $31.06^{* *}$ & $-24.51 * *$ \\
\hline 30 & PAIG 77 x PhuleDhanwantary & 134.31 & $7.60 *$ & -0.51 & $-15.49 * *$ & -5.32 & $-8.66 * *$ & 28.00 & $19.33^{*}$ & 17.25 & 17.25 & 19.15 & $-31.37 * *$ \\
\hline & S.E. \pm & 153.76 & 3.445 & 3.978 & 3.978 & 3.978 & 3.978 & 29.74 & 2.116 & 2.443 & 2.443 & 2.443 & 2.443 \\
\hline
\end{tabular}




\begin{tabular}{|c|c|c|c|c|c|c|c|c|c|c|c|c|c|}
\hline \multirow{3}{*}{$\begin{array}{l}\mathrm{Sr} \\
\text { no }\end{array}$} & \multirow[t]{3}{*}{ Hybrids } & \multicolumn{6}{|c|}{ Seed index $(\mathrm{g})$} & \multicolumn{6}{|c|}{ Lint index } \\
\hline & & \multirow[t]{2}{*}{ Mean } & \multirow{2}{*}{$\begin{array}{c}\text { M.P. } \\
\text { Heterosi } \\
\text { s }(\%)\end{array}$} & \multirow{2}{*}{$\begin{array}{c}\text { B.P. } \\
\text { Heterosi } \\
\text { s (\%) }\end{array}$} & \multicolumn{3}{|c|}{ \% standard heterosis over } & \multirow[t]{2}{*}{ Mean } & \multirow{2}{*}{$\begin{array}{c}\text { M.P. } \\
\text { Heterosis } \\
(\%)\end{array}$} & \multirow{2}{*}{$\begin{array}{c}\text { B.P. } \\
\text { Heterosis } \\
(\%)\end{array}$} & \multicolumn{3}{|c|}{ \% standard heterosis over } \\
\hline & & & & & PKVDH1 & $\begin{array}{c}\text { PKV } \\
\text { Suvarna }\end{array}$ & NACH 12 & & & & PKVDH1 & $\begin{array}{c}\text { PKV } \\
\text { Suvarna }\end{array}$ & $\begin{array}{c}\text { NACH } \\
12\end{array}$ \\
\hline 1 & PA $740 \times$ AKA 9703 & 5.34 & 14.36 & 5.64 & $22.50^{*}$ & 4.92 & $-17.16^{*}$ & 3.70 & 3.65 & 1.37 & 0.27 & 2.78 & 1.51 \\
\hline 2 & PA 740 x JLA 505 & 4.35 & -0.57 & -13.76 & 0.00 & -14.36 & $-32.38 * *$ & 3.53 & -0.35 & -2.08 & -4.07 & -1.67 & -2.88 \\
\hline 3 & PA $740 \times$ RAC 024 & 5.47 & $21.69^{*}$ & 8.32 & $25.60 *$ & 7.57 & $-15.06^{*}$ & 3.85 & $11.05^{* *}$ & $10.33 *$ & 4.34 & 6.95 & 5.63 \\
\hline 4 & PA $740 \times$ AKA 7 & 5.28 & -5.29 & -13.44 & 21.24 & 3.83 & $-18.01 *$ & 3.41 & -5.47 & $-8.58^{*}$ & -7.46 & -5.15 & -6.32 \\
\hline 5 & PA $740 \times$ PA 08 & 5.07 & 9.86 & 0.40 & 16.42 & -0.29 & $-21.27 * *$ & 3.50 & -0.50 & -1.41 & -5.02 & -2.64 & -3.85 \\
\hline 6 & PA 740 x PhuleDhanwantary & 4.49 & -3.34 & -11.09 & 3.10 & -11.70 & $-30.28 * *$ & 3.60 & 3.45 & 3.16 & -2.44 & 0.00 & -1.24 \\
\hline 7 & PA 760 x AKA 9703 & 5.07 & 18.87 & 18.46 & 16.42 & -0.29 & $-21.27 * *$ & 3.63 & 3.13 & -0.41 & -1.49 & 0.97 & -0.27 \\
\hline 8 & PA 760 x JLA 505 & 4.85 & $21.86^{*}$ & 14.12 & 11.37 & -4.62 & $-24.69 * *$ & 3.60 & 2.78 & -0.28 & -2.31 & 0.14 & -1.10 \\
\hline 9 & PA $760 \times$ RAC 024 & 5.37 & $31.14^{* *}$ & $26.35^{*}$ & $23.31^{*}$ & 5.60 & $-16.61 * *$ & 3.68 & $7.68^{*}$ & 6.98 & -0.14 & 2.36 & 1.10 \\
\hline 10 & PA $760 \times$ AKA 7 & 4.38 & -15.27 & $-28.11 * *$ & 0.69 & -13.77 & $-31.91 * *$ & 3.62 & 1.61 & -2.95 & -1.76 & 0.70 & -0.55 \\
\hline 11 & PA $760 \times$ PA 08 & 6.55 & $55.28^{* *}$ & $54.00 * *$ & $50.29 * *$ & $28.71^{* *}$ & 1.63 & 3.85 & $10.87^{* *}$ & $8.45^{*}$ & 4.48 & 7.09 & 5.77 \\
\hline 12 & PA 760 x PhuleDhanwantary & 4.54 & 6.83 & 6.71 & 4.13 & -10.82 & $-29.58 * *$ & 3.59 & 4.52 & 3.46 & -2.71 & -0.28 & -1.51 \\
\hline 13 & PA $848 \times$ x AKA 9703 & 6.55 & $51.68^{* *}$ & $50.46^{* *}$ & $50.29 * *$ & $28.71 * *$ & 1.63 & 4.00 & $13.54 * *$ & $9.88^{*}$ & $8.68^{*}$ & $11.40 * *$ & $10.03 *$ \\
\hline 14 & PA 848 x JLA 505 & 4.26 & 5.71 & -2.07 & -2.18 & -16.22 & $-33.85^{* *}$ & 3.60 & 2.42 & -0.42 & -2.44 & 0.00 & -1.24 \\
\hline 15 & PA $848 \times$ RAC 024 & 4.33 & 4.46 & -0.46 & -0.57 & -14.85 & $-32.76 * *$ & 3.62 & 5.55 & 5.09 & -1.90 & 0.56 & -0.69 \\
\hline 16 & PA 848 x AKA 7 & 4.31 & $-17.61 *$ & $-29.43 * *$ & -1.15 & -15.34 & $-33.15^{* *}$ & 3.54 & -0.84 & -5.09 & -3.93 & -1.53 & -2.75 \\
\hline 17 & PA $848 \times$ PA 08 & 5.33 & $24.97 *$ & $22.53 *$ & $22.39 *$ & 4.82 & $-17.24 *$ & 3.68 & 5.75 & 3.66 & -0.14 & 2.36 & 1.10 \\
\hline 18 & PA 848 x PhuleDhanwantary & 6.52 & $51.80^{* *}$ & $49.89 * *$ & $49.71^{* *}$ & $28.22 * *$ & 1.24 & 4.10 & $19.27 * *$ & $18.33 * *$ & $11.26^{* *}$ & $14.05^{* *}$ & $12.64 * *$ \\
\hline 19 & PA 828 x AKA 9703 & 5.28 & $27.72^{* *}$ & $23.25^{*}$ & 21.13 & 3.74 & $-18.09 *$ & 3.67 & 1.66 & 0.69 & -0.41 & 2.09 & 0.82 \\
\hline 20 & PA 828 x JLA 505 & 6.04 & $57.09 * *$ & $51.76^{* *}$ & $38.69 * *$ & $18.78^{*}$ & -6.21 & 3.67 & 2.16 & 1.66 & -0.41 & 2.09 & 0.82 \\
\hline 21 & PA 828 x RAC 024 & 6.13 & $54.92 * *$ & $54.15^{* *}$ & $40.87^{* *}$ & $20.65^{*}$ & -4.74 & 3.89 & $10.91^{* *}$ & $8.81^{*}$ & 5.56 & $8.21 *$ & 6.87 \\
\hline 22 & PA 828 x AKA 7 & 4.02 & $-20.24 *$ & $-34.10 * *$ & -7.69 & $-20.94 *$ & $-37.58 * *$ & 3.44 & -5.68 & -7.64 & -6.51 & -4.17 & -5.36 \\
\hline 23 & PA 828 x PA 08 & 4.48 & 9.80 & 7.18 & 2.87 & -11.90 & $-30.43 * *$ & 3.50 & -1.75 & -2.10 & -5.02 & -2.64 & -3.85 \\
\hline 24 & PA 828 x PhuleDhanwantary & 5.46 & $32.73^{* *}$ & $28.66^{*}$ & $25.26^{*}$ & 7.28 & $-15.30^{*}$ & 3.71 & 5.40 & 3.78 & 0.68 & 3.20 & 1.92 \\
\hline 25 & PAIG 77 x AKA 9703 & 6.55 & $53.51^{* *}$ & $53.15^{* *}$ & $50.52^{* * *}$ & $28.91^{* *}$ & 1.79 & 3.51 & -1.61 & -3.57 & -4.61 & -2.23 & -3.43 \\
\hline 26 & PAIG 77 x JLA 505 & 5.02 & $25.85^{*}$ & 17.72 & 15.15 & -1.38 & $-22.13 * *$ & 3.84 & $8.16^{*}$ & 6.51 & 4.34 & 6.95 & 5.63 \\
\hline 27 & PAIG 77 x RAC 024 & 4.63 & 12.93 & 8.69 & 6.31 & -8.95 & $-28.11 * *$ & 3.55 & 2.31 & 1.43 & -3.66 & -1.25 & -2.47 \\
\hline 28 & PAIG 77 x AKA 7 & 3.84 & $-25.87 *$ & $-37.05^{* *}$ & -11.83 & $-24.48^{*}$ & $-40.37 * *$ & 3.47 & -4.15 & -7.10 & -5.97 & -3.62 & -4.81 \\
\hline 29 & PAIG 77 x PA 08 & 5.06 & $19.79^{*}$ & 18.66 & 16.07 & -0.59 & $-21.51 * *$ & 3.67 & 4.26 & 3.52 & -0.27 & 2.23 & 0.96 \\
\hline \multirow[t]{2}{*}{30} & PAIG 77 x Phuledhanwantary & 5.16 & $21.41^{*}$ & 21.13 & 18.48 & 1.47 & $-19.88 * *$ & 3.61 & 3.52 & 3.00 & -2.17 & 0.28 & -0.96 \\
\hline & S.E. \pm & 4.952 & 0.401 & 0.463 & 0.463 & 0.463 & 0.463 & 3.621 & 0.121 & 0.140 & 0.140 & 0.140 & 0.140 \\
\hline
\end{tabular}


These branches provide structural arrangements or nodes for fruiting points which are finally converted into productive open bolls after floral shedding. As far as plant height is concerned, out of 30 crosses, the cross combination PA $828 \times$ PA 08 recorded highest significant positive heterosis over mid and better parent, while the cross combination PA $828 \times$ PA 08 recorded standard heterosis over three standard checks. These findings are in accordance with the results obtained by Dawod et al., (2010), Guvercin (2011), Patel et al., (2011), Jaiwar et al., (2012), Kumar et al., (2013).

For number of bolls per plant, positive heterosis is desirable. Out of 30 crosses, ten crosses displayed significant positive heterosis over PKVDH-1, while three crosses each exhibited significant positive heterosis over check PKV Suvarna. The cross combination PA 848 x PhuleDhanwantary exhibited maximum positive heterosis over mid parent, better parent and standard checks. Heterosis for this trait was reported by the earlier workers Tuteja et al., (2011), Balu et al., (2012) Jaiwar et al., (2012), Sekhar et al., (2012), Kumar et al., (2013) and Singh et al., (2013).

For the boll weight, positive heterosis is desirable. The cross combination PA $740 \mathrm{x}$ RAC 024 exhibited maximum positive heterosis over mid parent, better parent and standard checks PKVDH 1 and PKV Suvarna. Out of 30 crosses, three crosses were found superior over mid parent, three over better parent and two over standard check PKVDH1 and PKVSuvarna for boll weight. Heterosis for this trait was also reported by the earlier workers, Tuteja et al., (2011), Balu et al., (2012) Jaiwar et al., (2012), Sekhar et al., (2012) and Singh et al., (2013).

For the seed index, positive heterosis is desirable. Out of 30 crosses, fifteen crosses were found positively superior over mid parent, ten over better parent, eleven over standard check PKVDH 1 and six over standard check PKV Suvarna for seed index. The cross combination PA 828 x JLA 505 and PA 828 x RAC 024 exhibited maximum positive significant heterosis over mid parent, better parent respectively. The cross combination PAIG 77 x AKA 9703 exhibited maximum positive significant heterosis over standard check. Heterosis for this trait was reported by the earlier workers Khalid Hussain et al., (2009), Tuteja et al., (2011) and Balu et al., (2012).

For lint index heterosis in positive direction is desirable. The cross combination PA $848 \mathrm{x}$ Phuledhawantary exhibited maximum positive heterosis over mid parent and better parent. Out of thirty crosses, seven crosses recorded positive heterosis over mid parent, five over better parent and eight crosses over standard checks PKVDH 1. The crosses PA 848 x Phuledhawantary recorded highest significant positive heterosis over the checks PKVDH 1, PKV Suvarna and NACH 12. Similar results were obtained by Guvercin (2011).

The cross combination PA $848 \quad \mathrm{x}$ PhuleDhanwantary (121.59 \%) displayed significantly positive average heterosis for seed cotton yield per plant followed by PAIG 77 x AKA $9703(95.56 \%)$ and PA $760 \times$ PA 08 (84.17 \%). In case of better parent heterosis, the cross PA $848 \quad(118.59 \%)$ recorded highest significant positive heterosis followed by the crosses PAIG $77 \times$ AKA $9703(93.34 \%)$ and PA $760 \times$ PA $08(82.22$ $\%)$. The cross PA $848 \times$ PhuleDhanwantary displayed the highest significant positive heterosis over the standard check PKVDH 1 (118.59 \%), PKV Suvarna (122.23 \%) and NACH $12(27.94 \%)$. The range of heterosis over check PKV Suvarna was -26.67 per cent (PA $740 \times$ JLA 505) to 122.23 per cent (PA 
848 x Phule Dhanwantary). Heterosis for seed cotton yield and other related characters in arboreum cotton has also been reported earlier by Patel et al., 2010, Jaiwar et al., 2012, Kumar 2013 and Singh et al., 2013.

\section{References}

Balu, A.,P.D. Kavithamani, R. Ravikesavan, and S. Rajarathinam, (2012). Heterosis for seed cotton yield and its quantitative characters of Gossypium barbadense L. J. Cotton Res. and Dev., 26(1): 37-40.

Dawod, K.M. and A1-Guboory, K.K. 2010.Heterosis and combining ability in diallel cross among cultivars of upland cotton. Bulletin of Faculty of Agriculture, Cairo University., 61(1):1-7

Deosarkar, D. B. D. S. Jadhav, and S. G. Patil, (2009). Combining ability studies for yield and quality traits in cotton (Gossypium hirsutum L.). J.Cotton Res.Dev., 23(2):183-187.

Guvercin, R. S., (2011). Heterosis, heterobeltiosis and economic heterosis on some characters affecting fiber yields of F1cotton hybrids (Gossypium spp.). [Turkish]. Tarim Bilimleri Dergisi., 17(2): 113-121.

Jaiwar, S. S., H. A. Avinashe, and B. N. Patel, (2012). Heterosis for seed cotton yield and its contributing traits in upland cotton (G. hirsutum L.). J. Soils and Crops., 22(2): 314-320.

Khalid Hussain, Ghulam Abbas, Muhammad
Aslam, Hammad Hussnain, Akhtar, M. N. and Muhammad Irshad, (2009).Heterosis and inbreeding depression estimates for yield and fibre components in upland cotton $(G$. hirsutum L.). International J. Bio. Biotech., 6(4): 233-236.

Kumar A., (2013). Heterosis and combining ability for yield and fibre quality in desi cotton (Gossypium arboreumL.). M.Sc. Thesis submitted to V.N.M.K.V. Parbhani.

Patel, J. P., R. S. Fougat, G. C. Jadeja, C. G. Patel and K. P. Suthar, (2010). Heterosis study for yield and yield attributing character in inter-specific asiatic cotton hybrids. International $J$. Agri. Sci., 6(1): 78-83.

Patel, N. N., D. U. Patel, D. H. Patel, K. G. Patel, S. K. Chandran and V. Kumar, (2011). Study of heterosis in intervarietal crosses of Asiatic cotton (Gossypium herbaceum L.). World Cotton Research Conference-5, Mumbai, India, 149-152.

Singh, A., R. Avtar, R. K. Sheoran, A. Jain and G. Dharwal, (2013). Heterosis in male sterility based desi cotton hybrids for seed cotton yield and component traits. Annals of Biology., 29(1): 3234.

Tuteja, O. P., S. K. Verma and ManjuBanga (2011).Heterosis for seed cotton yield and other traits in GMS (Genetic male sterility) based hybrids of cotton (Gossypium hirsutum L.). J. Cotton Res. Dev., 25(1): 14-18.

\section{How to cite this article:}

Shinde, A.V., D.B. Deosarkar, V.N. Chinchane, A.S. Kalambe and N. Harshika. 2018. Study of Heterosis for Yield and Yield Contributing Traits in Desi Cotton (Gossypium arboreum L.). Int.J.Curr.Microbiol.App.Sci. 7(08): 4247-4255. doi: https://doi.org/10.20546/ijcmas.2018.708.445 